\title{
El coste del seguimiento farmacoterapéutico en una farmacia comunitaria (I): puesta en marcha del servicio
}

\author{
$M^{a}$ Belén Cobián Rodríguez \\ Doctora en Farmacia. Farmacéutica comunitaria en A Coruña.
}

\section{PALABRAS CLAVE}

seguimiento

farmacoterapéutico,

recursos, coste, servicios

en farmacia comunitaria

\section{ABREVIATURAS}

ZAP: zona de atención personalizada.

SFT: seguimiento

farmacoterapéutico.

PRM: problemas

relacionados con los

medicamentos.

RNM: resultados negativos

asociados a la medicación.

CIM: centro de

información de

medicamentos.

\section{RESUMEN}

Introducción: La gestión de un servicio de seguimiento farmacoterapéutico (SFT) requiere la planificación de los recursos que se van a utilizar para alcanzar los objetivos que se han determinado. El objetivo de este trabajo fue estudiar los recursos necesarios para la puesta en marcha del servicio de SFT en una farmacia comunitaria y su coste.

Material y métodos: En la farmacia Cobián se identificaron los recursos materiales, humanos y de información necesarios para la puesta en marcha del servicio, se evaluaron y se proveyeron los necesarios. Se diseñó un programa de formación en seguimiento farmacoterapéutico y se estudió el tiempo dedicado a formación y el coste de esta. Se solicitó la acreditación de la formación impartida.

Resultados: No fue necesaria la provisión de recursos materiales. Como recurso de información a proveer se identificó la historia clínica del paciente si bien esto no supuso coste alguno. El tiempo total de formación de las dos farmacéuticas fue de 206 horas con un coste asociado de 1.900 euros cada una. Fue acreditada con 9,2 créditos.

Discusión: Las zonas de atención personalizada de la farmacia cumplieron todos los requisitos. La historia clínica fue fundamental para la realización del SFT debería ponerse a disposición del farmacéutico siempre que lo autorizara el paciente. La formación supuso 1.900 euros por farmacéutica, si bien el coste de formación por paciente disminuirá al aumentar el número de estos. Se consiguió la acreditación externa de la formación.

The cost of pharmacotherapy follow in a community pharmacy (I) service launch

\section{ABSTRACT}

Introduction: Managing a pharmacotherapy follow-up service requires planning the resources to be used in reaching the objectives predetermined. The aim of this work was to study the resources needed for the implementation of a pharmacotherapy follow-up service in a community pharmacy and their costs.

Methods: In pharmacy Cobián, resources for the implementation of the service were identified, evaluated and provided. A training program in pharmacotherapy follow-up was designed and training time and its cost were studied. External training accreditation was applied.

Results: It was not necessary to provide material resources. Patient's clinical history was the only information resource that had to be provided though it had no associated cost. Training time for the two pharmacists was 206 hours and its cost was $1.900 €$ for each one. Training program was accredited with 9.2 points.

Discussion: Personalized care areas in the pharmacy met all requirements. Patient's clinical history is essential and it ought to be available for pharmacists in the service Training costs per patient will decrease on increasing the number of patients in the program.

El estudio ha sido realizado con datos recogidos en la elaboración de la tesis doctoral de la autora: Diseño, implantación y análisis de un modelo para la gestión del seguimiento farmacoterapéutico en una farmacia comunitaria. Los costes del seguimiento farmacoterapéutico han sido actualizados a mayo de 2013.

Parte de los datos expuestos se han utilizado en la ponencia Gestión del seguimiento farmacoterapéutico en una farmacia comunitaria: Formación de los profesionales. II Congreso Nacional de Farmacéuticos Comunitarios (SEFAC) celebrado en A Coruña 2006.

Financiación: Ninguna ajena.

Conflicto de intereses: La autora declara no existir ningún conflicto de intereses en relación con el contenido del presente articulo. Cite este artículo como: Cobián MB. El coste del seguimiento farmacoterapéutico en una farmacia comunitaria (I): puesta en marcha del servicio. Farmacéuticos Comunitarios. 2014 Jun 01;6(2):27-32. doi:10.5672/FC.2173-9218.(2014/Vol6).002.05

Correspondencia: Ma Belén Cobián Rodriguez (farmaciacobian@gmail.com). 


\section{Introducción}

La gestión de un servicio requiere, entre otras actividades, la planificación de los recursos que se van a utilizar para alcanzar las metas y los objetivos que se han determinado (1). Dicha planificación comprende la identificación de los recursos que se utilizaran y la previsión de utilización de los mismos, la evaluación de los recursos de los que se dispone y la provisión de aquellos que sean necesarios como consecuencia de la evaluación.

El seguimiento farmacoterapéutico (SFT) ha sido definido como "el servicio profesional que tiene como objetivo la detección de problemas relacionados con los medicamentos (PRM) para la prevención y resolución de resultados negativos asociados a la medicación (RNM). Este servicio implica un compromiso y debe proveerse de forma continuada, sistematizada y documentada, en colaboración con el propio paciente y con los demás profesionales del sistema de salud, con el fin de alcanzar resultados concretos que mejoren la calidad de vida del paciente" (2).

El SFT requiere cambios en la estructura habitual de la farmacia. $\mathrm{Al}$ estar basado en una relación terapéutica con el paciente se hace necesario un entorno que garantice la confidencialidad. Se utilizan para ello las llamadas Zonas de Atención Personalizada (ZAP), que constituyen lugares dentro de la farmacia que proporcionan áreas privadas 0 semiprivadas para el ejercicio de la atención farmacéutica. Las características que deben reunir las ZAP han sido descritas por varios autores. Así Cipolle y col describieron las condiciones que deben reunir los lugares en los que se realiza el SFT (3). En España, Aguiló definió y caracterizó las ZAP (4), señalando su equipamiento mínimo.

Pero además, el SFT precisa de unos recursos de información. El conocimiento de las patologías que presenta el paciente y su relación con los medicamentos que utiliza constituye una información básica en la realización de este servicio. Para ello, además de conocer los medicamentos que utiliza el paciente, lo cual se puede conseguir mediante registros electrónicos de la medicación dispensada en la farmacia, es importante tener acceso a información contenida en la historia clínica del paciente en cuanto a diagnósticos y pruebas analíticas. Esta información puede ser proporcionada por el propio paciente o bien por otros profesionales que le atienden con su autorización. Una vez obtenida la información es necesario evaluar su farmacoterapia y para ello se utilizarán las fuentes de información de medicamentos y en caso de evaluaciones especialmente complejas será necesaria una ayuda especializada que puede obtenerse de los centros de información de medicamentos.

Por otra parte, al tratarse de un servicio profesional, el SFT necesita farmacéuticos específicamente formados para realizarlo. Por lo tanto, la formación y el tiempo de estos profesionales se presuponen importantes para la puesta en funcionamiento de este servicio.

En la planificación de un servicio de SFT en una farmacia comunitaria nos planteamos como objetivo evaluar los recursos de que disponíamos para implantar el servicio y proveer aquellos que fuesen necesarios, estudiando su coste. En concreto, en este artículo se estudian los costes de la puesta en marcha del servicio.

\section{Material y métodos}

El estudio se realizó en la farmacia Cobián de A Coruña. Se identificaron los recursos necesarios para la puesta en marcha del servicio de SFT observando aquellos que se utilizaron para el SFT de un solo paciente. Se valoraron los recursos que fue necesario proveer así como el consumo de aquellos de los que ya disponía la farmacia por utilizarse en otros servicios.

\section{Recursos materiales}

Zona de Atención personalizada (ZAP). Siguiendo a Aguiló (4), los criterios utilizados para evaluar la ZAP fueron: visibilidad desde la zona de dispensación, accesibilidad, diferenciación del mostrador y otros elementos de la zona de dispensación, privacidad para la comunicación farmacéutico-paciente y un equipamiento mínimo, número de ZAP y zona de espera para los pacientes que deben ser atendidos.

\section{Recursos de información}

Registros electrónicos, en concreto fichas de registro de la medicación, que son documentos informáticos que registran el perfil de utilización de medicamentos por los pacientes. Información procedente de otros profesionales sanitarios, que complementen la historia farmacoterapéutica. Información de medicamentos, tanto por consultas a fuentes bibliográficas como por consultas a Centros de Información de Medicamentos (CIM)

\section{Recursos humanos}

En la farmacia trabajaban dos farmacéuticas adjuntas y una técnico en farmacia, además de la farmacéutica titular. Solo esta última tenía experiencia previa en SFT. Se identificó la necesidad de formación de las farmacéuticas adjuntas y de la provisión de tiempo para realizarla.

Se diseñó un programa de formación en seguimiento farmacoterapéutico (Anexo I) que fue impartido por la farmacéutica titular, siendo las dos farmacéuticas adjuntas discentes.

Al impartirse el curso en la farmacia, las variables que determinaron el coste del programa de formación fueron los tiempos invertidos por las farmacéuticas en las distintas fases y sus costes asociados.

Para determinar el coste del tiempo de las farmacéuticas utilizamos el convenio vigente en mayo de 2013, lo que, unido al coste de la seguridad social, supuso un coste por hora trabajada de 18,53 euros.

Los registros se realizaron utilizando las hojas de registro del método Dáder (5) y una hoja de registro de recursos diseñada al efecto.

Para valorar la calidad de la formación impartida se solicitó su acreditación al Sistema Acreditador de la Formación Continuada de las Profesiones Sanitarias de la comunidad autónoma de Galicia.

\section{Resultados}

\section{Recursos materiales}

La farmacia disponía de dos Zonas de Atención Personalizada que según la clasificación de Aguiló (4) se correspondian con una zona tipo I, ZAP cercana a la zona de dispensación y una tipo III, ZAP separada 
de la zona de dispensación, ubicada en un despacho interior. Ambas resultaron estar equipadas con todo lo necesario, por lo que no fue necesaria ninguna inversión. Las características consideradas están recogidas en la tabla 1.

\section{Recursos de información}

Registros electrónicos

Existian fichas de registro de la medicación utilizada por los enfermos crónicos, usuarios habituales de la farmacia que fueron utilizadas en todos los casos.

\section{Información procedente de otros}

profesionales

- Informes médicos y pruebas analíticas, fueron aportados por los pacientes y permitieron conocer sus diagnósticos y estado de salud. No supusieron ningún coste adicional.

- Entrevistas personales utilizando tanto el teléfono como los informes escritos e incluso a través del paciente. No supusieron ningún coste apreciable dentro del necesario para el funcionamiento normal de la farmacia.

\section{Información de medicamentos}

En el transcurso del programa de formación se utilizaron:

- Fuentes bibliográficas: la base de datos de medicamentos del Consejo General de Colegios Oficiales de Farmacéuticos (6), la Guía sobre el diagnóstico y el tratamiento de la hipertensión arterial en España (7), el Tratado de medicina interna de Harrison (8) y Diagnóstico clínico por el laboratorio de Todd-Sandford" (9). No fue necesario ampliar la bibliografía ya existente en la farmacia.

- Centros de Información de Medicamentos: se realizaron consultas al Centro de Información de Medicamentos del Colegio Oficial de Farmacéuticos de A Coruña. $\mathrm{Al}$ estar incluido el servicio en la cuota de colegiación las consultas no supusieron un coste adicional.

- Es decir, en todos los casos se trató de recursos de información de los que ya disponía la farmacia o bien fueron aportados por los propios pacientes y no supusieron un coste añadido.
Tabla 1 Características de la Zona de Atención Personalizada

\begin{tabular}{|l|c|}
\hline Visibilidad desde la zona de dispensación & Si \\
\hline Accesibilidad. & $\mathrm{Si}$ \\
\hline Diferenciación del mostrador de la zona de dispensación & $\mathrm{Si}$ \\
\hline Privacidad para la comunicación farmacéutico-paciente & $\mathrm{Si}$ \\
\hline Zona de espera para los pacientes de la ZAP & $\mathrm{Si}$ \\
\hline Equipamiento mínimo: & \\
\hline - Una mesa amplia y dos sillas & $\mathrm{Si}$ \\
\hline - Una biblioteca de consulta básica & $\mathrm{Si}$ \\
\hline - Acceso informático a bases de datos e internet & $\mathrm{Si}$ \\
\hline - Teléfono y fax & $\mathrm{Si}$ \\
\hline - Herramientas de apoyo: dispositivos de prueba, protocolos, hojas de informe, registros, etc. & $\mathrm{Si}$ \\
\hline - Aparataje, tensiómetro, cronómetro, glucómetro y báscula & $\mathrm{Si}$ \\
\hline
\end{tabular}

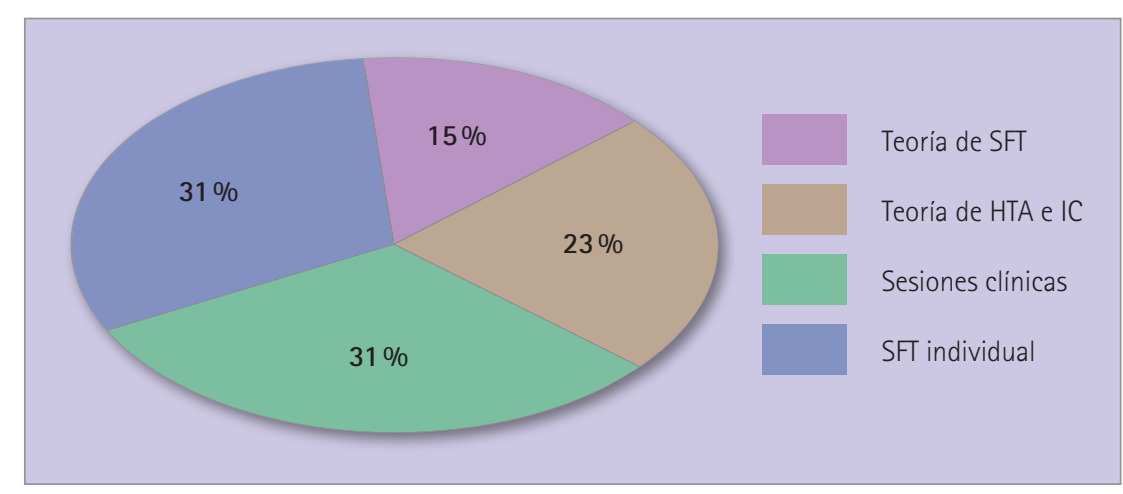

Figura 1 Distribución del tiempo del programa de formación en SFT

\section{Recursos humanos}

Tiempo del periodo de formación

El recurso que se utilizó en mayor medida fue el tiempo de los farmacéuticos. Se dedicaron a formación 206 horas, repartidas de la siguiente forma:

- La formación teórica ocupó un total de 77 horas, resultantes de la suma del tiempo dedicado a la metodología del SFT, 30 horas, y del tiempo dedicado a la formación teórica en las patologías seleccionadas, 47 horas.

- La parte práctica del programa ocupó un total de 129 horas, de las cuales se corresponden con las sesiones clínicas 63 horas. El tiempo de seguimiento farmacoterapéutico individual tutelado fue de 66 horas.

Todo ello está representado en la figura 1.

Dentro de la parte práctica de formación en seguimiento farmacoterapéutico, el número de veces que se realizó cada actividad, el tiempo empleado en cada una de ellas y sus costes, están reflejados en la tabla 2.

\begin{tabular}{|l|c|l|}
\hline Actividad & Número & Tiempo \\
\hline Sesiones clinicas & 7 & 63 horas \\
\hline Entrevista inicial & 16 & 9 horas y 15 minutos \\
\hline Estado de situación & 42 & 9 horas \\
\hline Evaluación global & 42 & 29 horas \\
\hline Intervención farmacéutica & 33 & 6 horas y 30 minutos \\
\hline Visitas & 68 & 12 horas y 35 minutos \\
\hline
\end{tabular}

Tabla 2 Distribución del tiempo y costes de cada fase del programa de formación 
Tabla 3 Costes del programa de formación

\begin{tabular}{|l|c|}
\hline Actividad & Coste $(\epsilon)$ \\
\hline $\begin{array}{l}\text { Formación teórica en } \\
\text { metodología de SFT }\end{array}$ & 556 \\
\hline $\begin{array}{l}\text { Formación teórica en } \\
\text { patologias prevalentes }\end{array}$ & 871 \\
\hline Sesiones clínicas & 1.167 \\
\hline SFT individual tutelado & 1.223 \\
\hline Total Programa de Formación & 3.817 \\
\hline
\end{tabular}

El coste total del programa de formación de las dos farmacéuticas fue de $3.817 €$, que se corresponden con los costes parciales especificados en la tabla 3 .

\section{Acreditación externa de la actividad formativa}

- La acreditación se solicitó estimando en cincuenta horas por farmacéutico el tiempo dedicado a la parte práctica. En realidad, se emplearon cincuenta y una horas en un caso y cincuenta y seis en el segundo.

- El sistema acreditador de la formación continuada de las profesiones sanitarias de la comunidad autónoma de Galicia acreditó la actividad asignándole 9,2 créditos.

\section{Discusión}

Se identificaron los recursos necesarios para poner en marcha un servicio de SFT en una farmacia comunitaria estudiando los recursos necesarios para la atención de un solo paciente. En la farmacia se venía realizando con anterioridad el SFT de algunos pacientes por lo que el trabajo se desarrolló para transformar esa atención a algunos pacientes en un servicio organizado.

En la evaluación de los recursos materiales, se comprobó que las dos ZAP de las que disponía la farmacia estaban suficientemente equipadas por lo tanto no fue necesario actuar sobre las mismas. La existencia de una ZAP es imprescindible para el servicio de SFT, por lo tanto una farmacia que no disponga de ella tendrá que habilitarla. El coste que esto puede suponer es muy variable en función de las características estructurales de cada farmacia en particular.

En la evaluación de los recursos de información se puso de manifiesto la importancia de los registros informáticos de la medicación y de disponer de datos clínicos del paciente para poder realizar la evaluación de su medicación. El acceso a la historia clínica es importante para proporcionar al farmacéutico toda la información necesaria (10). Ha de ser aportada por el paciente o con su consentimiento y el acceso a esta información es necesario para la inclusión del paciente en el servicio.

Por otra parte, la bibliografía existente en la farmacia fue suficiente y las consultas al CIM del colegio de A Coruña completaron el estudio en los casos necesarios, todo ello sin suponer un coste atribuible específicamente al SFT.

En la evaluación de los recursos humanos resultó evidente la necesidad de formación de las farmacéuticas adjuntas. Dicha formación fue lo que realmente concentró la inversión necesaria para poner en funcionamiento el servicio. Este hecho era esperado puesto que al tratarse de un servicio profesional la preparación y el tiempo de las farmacéuticas resulta imprescindible. Por otra parte, la necesidad de formación se pone de manifiesto cuando se estudian las barreras o limitaciones a la implantación del SFT (11-14).

La formación se realizó en la farmacia diseñando un programa teórico-practico (Anexo I) basado en el método Dáder e incluyendo formación en las patologías que esperábamos fuesen las más prevalentes, y que se determinaron en un estudio realizado en la farmacia (15).

El tiempo invertido en el proceso de formación de las dos farmacéuticas fue de 206 horas, resultantes de la suma de 77 horas de formación teórica con 129 horas de formación práctica. Es discutible el número mínimo de casos prácticos que debe realizar un profesional para estar preparado para realizar el SFT puesto que depende de varios factores no controlables como los conocimientos y habilidades previas del farmacéutico, la casuística que presenten los pacientes y el propio método de formación que permite aprender a medida que se realiza el proceso de SFT.

A la hora de valorar el coste de la formación hay que tener en cuenta que esta fue impartida por el titular de la farmacia. En caso de que fuese necesaria una formación externa, debería añadirse al tiempo invertido el coste de la formación recibida y en su caso los desplazamientos. El coste de la formación en las circunstancias señaladas resultó ser de unos 1.900 euros por farmacéutica. Para valorar dicho coste tendremos que tener en cuenta que se trata de un gasto fijo, es decir el coste por paciente se irá haciendo proporcionalmente menor a medida que aumente el número de pacientes.

Por otra parte, se consideró imprescindible la acreditación externa de la actividad formativa. Se obtuvo la citada acreditación del Sistema Acreditador de la Formación Continuada de las Profesiones Sanitarias de Galicia, que lo valoró con 9,2 créditos. Creemos que dicha valoración no se ajusta ni al tiempo ni al esfuerzo invertidos pero hay que señalar que es la primera vez que dicho sistema reconoce la actividad formativa de la farmacia comunitaria y a esta como centro de formación de postgrado.

En resumen, los recursos que fue necesario aportar al sistema para la puesta en marcha del servicio de SFT fueron la información sobre el estado de salud de los pacientes y la formación en SFT de las farmacéuticas adjuntas. La información no supuso ningún coste mientras que la formación tuvo un coste de 1.900 euros por farmacéutica. 


\section{Anexo 1. Formación en seguimiento farmacoterapéutico}

\section{Objetivo general}

- Capacitar a las farmacéuticas adjuntas para realizar el seguimiento farmacoterapéutico de pacientes en una farmacia comunitaria.

\section{Objetivos específicos}

Que las farmacéuticas participantes:

- Adquieran conocimientos de metodología de seguimiento farmacoterapéutico.

- Actualicen su conocimiento de los tratamientos de las patologías más prevalentes entre los pacientes de la farmacia: hipertensión e insuficiencia cardiaca.

- Sean capaces de realizar la exposición y discusión de los casos en sesiones clínicas.

- Desarrollen habilidades de comunicación en entrevistas farmacéutico paciente.

\section{Participantes}

Farmacéuticas adjuntas.

\section{PROGRAMA DE FORMACIÓN}

\section{A. Programa teórico}

Formación teórica en enfermedades más prevalentes en la farmacia: las enfermedades que consideramos como más prevalentes en la farmacia fueron hipertensión arterial e insuficiencia cardiaca.

\section{Insuficiencia cardiaca}

- Diagnóstico de la insuficiencia cardiaca crónica: Epidemiología, fisiopatología y métodos diagnósticos.

- Tratamiento de la insuficiencia cardiaca:

- Objetivos, prevención de la insuficiencia cardiaca, manejo no farmacológico.

- Tratamiento farmacológico:

I. Inhibidores de la enzima conversora de angiotensina, diuréticos, antagonistas de los receptores beta adrenérgicos, antagonistas de los receptores de la aldosterona, antagonistas de los receptores de la angiotensina II, glucósidos cardíacos, agentes vasodilatadores, agentes antitrombóticos y antiarrítmicos.

II. Elección y programación del tratamiento farmacológico.

\section{Hipertensión arterial}

- Definición. Epidemiología. Causas.

- Medida de la presión arterial.

- Evaluación clínica del paciente hipertenso.

- Objetivos generales del tratamiento.

- Tratamiento no farmacológico.

- Tratamiento farmacológico:

- Principios del tratamiento.

- Fármacos antihipertensivos: diuréticos, betabloqueantes, calcioantagonistas, inhibidores del enzima de conversión de la angiotensina, antagonistas de los receptores de angiotensina II, alfabloqueantes y otros fármacos antihipertensivos.

- Tratamiento farmacológico combinado.

B. Formación teórica en seguimiento farmacoterapéutico El programa que se realizó en cinco sesiones de dos horas cada una de acuerdo con el siguiente esquema:

Primera sesión:

- Problemas relacionados con los medicamentos, concepto y definición.

- Clasificación de los PRM.

- Morbilidad relacionada con medicamentos, importancia en salud pública.

Segunda sesión:

- El programa Dáder de seguimiento farmacoterapéutico: objetivos y definiciones.

- Técnicas de comunicación en seguimiento farmacoterapéutico.

Tercera sesión:

- La entrevista inicial.

- Elaboración del estado de situación del paciente.

- Fase de estudio del caso: Procedimiento. Búsquedas bibliográficas.

Cuarta sesión:

- Evaluación y plan de actuación.

- Intervenciones farmacéuticas.

- Registro de visitas y evaluaciones posteriores.

Quinta sesión:

- Estándar de elaboración y exposición de casos en seguimiento farmacoterapéutico. Modelo a seguir. Ejemplos.

- Elaboración y exposición de los casos por las farmacéuticas participantes en tiempo máximo de una hora y siguiendo el modelo.

\section{Programa práctico}

Sesiones clínicas

- La farmacéutica responsable del paciente, realiza el siguiente trabajo:

- Exposición del Estado de Situación, resultado de la Evaluación Global y propuesta de un Plan de Actuación para cada paciente incorporado al programa o para aquellos que hayan sufrido una modificación en su estado de salud o tratamiento farmacológico.

- Exposición y discusión de los resultados de las Intervenciones Farmacéuticas realizadas durante la semana.

Seguimiento farmacoterapéutico individual: cada farmacéutica realizó seguimiento farmacoterapéutico de ocho pacientes siguiendo el método Dáder. 


\section{Referencias bibliográficas}

1. Schumock GT, Wong G. Business planning for pharmacy programs. In: Deselle SP, Zgarrick D, editors. Pharmacy management. Essentials for all practice settings. New York: McGraw-Hill; 2005. p. 79-94.

2. Foro de Atención Farmacéutica. Documento de consenso. Madrid: Consejo General de Colegios Oficiales de Farmacéuticos; 2008.

3. Cipolle RJ, Strand LM, Morley PC. Pharmaceutical care practice. New York; MacGraw-Hill: 1998.

4. Aguiló M. Estructura física de la farmacia. En: Faus MJ, Martínez F, editores. Diploma de Atención Farmacéutica. Valencia: General Asde; 1999. p. 71-85.

5. Sabater D, Silva MM, Faus MJ. Método Dáder. Guía de Seguimiento Farmacoterapéutico. Tercera edición. Granada: Grupo de Investigación en Atención Farmacéutica (CTS-131): Universidad de Granada; 2007.
6. Consejo General de Colegios Oficiales de Farmacéuticos. Base de datos del conocimiento sanitario. [CD-Rom]. Madrid: CGCOF; 2005.

7. Sociedad Española de Hipertensión, Liga Española de Lucha contra la Hipertensión Arterial (SEH-LELHA). Guía sobre el diagnóstico y tratamiento de la hipertensión arterial en España 2002. Hipertensión. 2002;19(3):1-74.

8. Harrison. Principios de Medicina Interna. Madrid: Mc-Graw-Hill-Interamericana; 1994.

9. Davidson I, Henry JB. Diagnóstico clinico por el laboratorio. Sexta edición. Barcelona: Todd-Sandford; 1978.

10. Baena I, Martínez J, Faus MJ, Martínez F. Seguimiento farmacoterapéutico integral de pacientes en el sistema sanitario. Pharm Care Esp. 2002;4:325-332.

11. Zardain E. Factores psicosociales y proceso de cambio en farmacéuticos comunitarios españoles [tesis]. Oviedo: Universidad de Oviedo; 2007.

12. Van Mill J, De Boer W, Tromp T. European barriers to the implemen- tation of pharmaceutical care. Int J Pharm Pract. 2001;9:163-168. doi:10.1111/j.2042-7174.2001.tb01044.x

13. Gastelorrutia MA, Fernández-Llimós F, García-Delgado P, Gastelurrutia P, Faus MJ, Benrimoj SI. Barreras y facilitadores para la diseminación e implantación de servicios cognitivos de la farmacia comunitaria española. Seguimiento Farmacoterapéutico. 2005;3(2):65-77.

14. Andrés Iglesias JC, Rodríguez Sampedro A, Cobián Rodríguez B, Brizuela Rodicio L, Fornos Pérez JA, Andrés Rodríguez NF. Barreras para la implantación del seguimiento farmacoterapéutico en Galicia. [Internet]. Comunicación Simpodáder Murcia 2006. [acceso 12/1/2014]. Disponible en: http://grupoberbs.weebly.com/ uploads/2/6/0/1/26011151/barreras. png

15. Cobian B, Lourés P. Planificación de los servicios de atención farmacéutica para los pacientes mayores en una farmacia comunitaria. e-farmacéutico comunitario. 2006; [extra]:34. 